\title{
Latent Transforming Growth Factor-beta 1 Functionalised Electrospun Scaffolds Promote Human Cartilage Differentiation: Towards an Engineered Cartilage Construct
}

\author{
Erh-Hsuin Lim ${ }^{1,2}$, Jose Paulo Sardinha ${ }^{1,3}$, Simon Myers ${ }^{2}$, Molly Stevens ${ }^{1}$ \\ ${ }^{1}$ Department of Materials and Institute of Biomedical Engineering, Imperial College London, London; ${ }^{2}$ The Blizard Institute of Cell and \\ Molecular Science, Barts and The London School of Medicine and Dentistry, Queen Mary University of London, London, UK; ${ }^{3}$ Instituto \\ Superior Técnico and ICEMS, Lisbon, Portugal
}

Background To overcome the potential drawbacks of a short half-life and dose-related adverse effects of using active transforming growth factor-beta 1 for cartilage engineering, a cell-mediated latent growth factor activation strategy was developed incorporating latent transforming growth factor- $\beta 1$ (LTGF) into an electrospun poly(L-lactide) scaffold.

Methods The electrospun scaffold was surface modified with $\mathrm{NH}_{3}$ plasma and biofunctionalised with LTGF to produce both random and orientated biofunctionalised electrospun scaffolds. Scaffold surface chemical analysis and growth factor bioavailability assays were performed. In vitro biocompatibility and human nasal chondrocyte gene expression with these biofunctionalised electrospun scaffold templates were assessed. In vivo chondrogenic activity and chondrocyte gene expression were evaluated in athymic rats.

Results Chemical analysis demonstrated that LTGF anchored to the scaffolds was available for enzymatic, chemical and cell activation. The biofunctionalised scaffolds were non-toxic. Gene expression suggested chondrocyte re-differentiation after 14 days in culture. By 6 weeks, the implanted biofunctionalised scaffolds had induced highly passaged chondrocytes to re-express Col2A1 and produce type II collagen.

Conclusions We have demonstrated a proof of concept for cell-mediated activation of anchored growth factors using a novel biofunctionalised scaffold in cartilage engineering. This presents a platform for development of protein delivery systems and for tissue engineering.

Keywords Transforming growth factor beta1 / Guided tissue regeneration / Biomimetics / Tissue scaffolds / Cartilage
Correspondence: Erh-Hsuin Lim The Blizard Institute of Cell and Molecular Science, Barts the London School of Medicine and Dentistry, Queen Mary University of London, 4 Newark Street, London E1 2AT, UK Tel: +44-2078822381

Fax: +44-2078827172 E-mail: ehlim28@gmail.com

The concept of this article has been granted an international (WO/2009/144457) and a US patent (US20110123592).

This article was presented at the Society of Academic Research Surgery on Jan 6, 2010 in London, UK and BAPRAS Winter Scientific Meeting on December 5, 2008 in London, UK. This study was awarded the Gibson Prize for the best paper presented at SARS 2010.

No potential conflict of interest relevan to this article was reported.

\section{INTRODUCTION}

Growth factors provide key bioactive cues in vivo and are im- portant for tissue engineering strategies. However, their short half-lives and uncontrolled dose-related adverse effects present challenges [1]. Attempts have been made to incorporate growth

Copyright ( 2013 The Korean Society of Plastic and Reconstructive Surgeons

This is an Open Access article distributed under the terms of the Creative Commons Attribution Non-Commercial License (http://creativecommons.org/

licenses/by-nc/3.0/) which permits unrestricted non-commercial use, distribution, and reproduction in any medium, provided the original work is properly cited. I www.e-aps.org 
factor delivery into scaffolds [1]. Transforming growth factor beta 1 (TGF- $\beta 1$ ) has a broad spectrum of chondrogenic roles [2] relevant to cartilage engineering specifically. In native cartilage extracellular matrix (ECM), TGF- $\beta 1$ is secreted by chondrocytes in its latent form [3]. The latent TGF- $\beta 1$ (LTGF) consists of a mature (active) TGF- $\beta 1$ associated to its pro-peptide, the latency-associated peptide (LAP), by non-covalent interactions [4]. LTGF is then bound to the LTGF binding protein (LTBP), itself covalently anchored to the ECM. This complex formation between LTGF and LTBP is mediated by an intramolecular disulphide exchange between the third 8-cysteine (8-Cys-3) domain of the LTBP and a pair of cysteine-33 (Cys33) residues of LAP (Fig. 1) [5]. The LAP-TGF- $\beta 1$ association preserves the bioactivity of the growth factor [6]. The half-life of the LTGF of rats has been reported to be greater than 100 minutes as compared with a short 2 to 3 minutes for the active form [7]. The activation/release of TGF- $\beta 1$ involves disruption of the non-covalent interaction between the LAP and TGF- $\beta 1$ by proteases, thrombospondin-1, low $\mathrm{pH}$ and reactive oxygen species $[4,8]$. The LAP also possesses two arginine-glycineaspartic acid sequences which activate the TGF- $\beta 1$ on cell attachment [4]. In general, TGF- $\beta 1$ stimulates growth in mesenchymal cells and is inhibitory to epithelial and endothelial cells [8]. The cell response to TGF- $\beta 1$ varies depending on the dose, length of exposure and target tissue [8]. A prolonged exposure and high dose of TGF- $\beta 1$ may result in pathological conditions such as extensive fibrosis, hypertrophic scarring and formation of osteophytes [8,9]. An engineered system capable of inducing cell-mediated TGF- $\beta 1$ activation will begin to address these problems through cartilage engineering. The novel strategy presented in this study is to incorporate LTGF onto an electrospun scaffold and allow chondrocytes to sense and activate TGF- $\beta 1$

\section{Fig. 1. A schematic representation of LTGF in the ECM}

The LAP-TGF- $\beta 1$ association (RCSB Protein Databank-1KLC) is attached to the LTBP, which is anchored to the ECM [4]. RCSB, Research Collaboratory for Structural Bioinformatics; LAP, latency-associated peptide; TGF, transforming growth factor; LTGF, latent transforming growth factor- $\beta 1$; LTBP, LTGF binding protein; ECM, extracellular matrix.

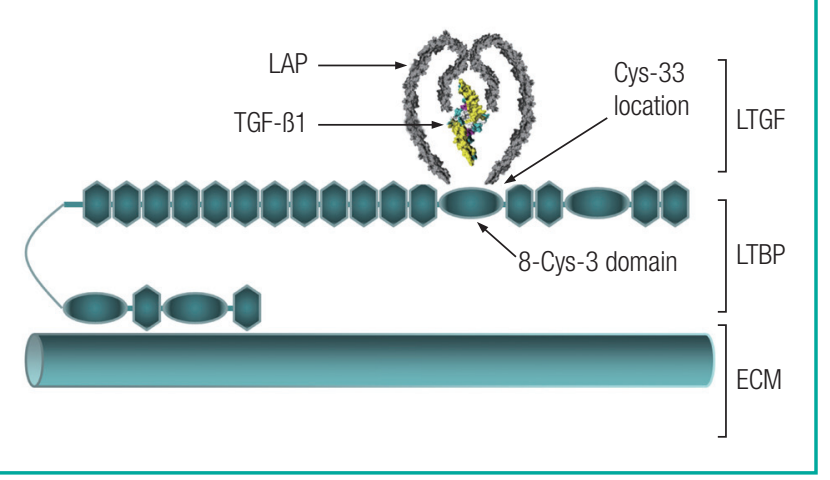

as required. Two techniques of scaffold biofunctionalisation are described: scaffold surface modification using ammonia plasma, and this combined with subsequent sulfosuccinimidyl-4- $(N-$ maleimidomethyl)cyclohexane-1-carboxylate (sulfo-SMCC) treatment. The effects of scaffold biofunctionalisation with LTGF on the cell phenotype were evaluated in vitro and in vivo using primary human nasal chondrocytes.

\section{METHODS}

\section{Scaffold fabrication by electrospinning}

3.0\% (w/w) poly(L-lactide) (PLLA, Purac Biochem, Birmingham, UK), dissolved in dichloromethane/dimethylformamide (70:30 [w/w], AGTC Bioproducts, Loughborough, UK) was electrospun at a feed rate of $0.4 \mathrm{~mL} / \mathrm{hr}$, accelerating voltage of $10 \mathrm{kV}$ and $10 \mathrm{~cm}$ tip-to-collector distance. The resulting scaffold was sterilised by ultraviolet irradiation (30 minutes), and rinsed in $70 \%(\mathrm{v} / \mathrm{v})$ ethanol.

\section{Biofunctionalisation of scaffold}

Surface modification of electrospun scaffolds by plasma treatment

The scaffold was treated with ammonia gas $\left(\mathrm{NH}_{3}\right)$ plasma using Plasprep 5 (GaLa Instrumente, Bad Schwalbach, Germany). The treatment process was performed at a range of exposure times (2, 5, and 10 minutes) and powers (10-100 W), while the pressure was kept constant at $2.0 \mathrm{mbar}$. An exposure time of 10 minutes at $100 \mathrm{~W}$ was chosen for the experiment.

\section{Biofunctionalisation of scaffold with latent TGF- $\beta 1$ complex} After plasma treatment (10 minutes, $100 \mathrm{~W}$ ), two LTGF immobilisation techniques were performed: 1) "random immobilisation" where the LTGF was anchored to the scaffold at non-specific sites of the LAP; and 2) "orientated immobilisation” where sulfo-SMCC (Sigma, Gillingham, UK) was used to crosslink the LTGF at the Cys-33 residue of the LAP (using its $\mathrm{N}$-hydroxysuccinimide terminal) to the scaffold surface amine groups (with the maleimide terminal) (Fig. 2).

In the random immobilisation technique, the plasma-treated scaffold was immediately immersed in recombinant human latent TGF- $\beta 1$ solution $(0.75 \mu \mathrm{g} / \mathrm{mL}, \mathrm{R} \& \mathrm{D}$ Systems, Abingdon, UK) for 2 hours at room temperature (Fig. 2). The scaffold was then rinsed $(\times 3)$ in sterile phosphate-buffered solution (PBS, Gibco, Paisley, UK).

In the orientated immobilisation technique, the plasma-treated scaffold was rinsed $(\times 3)$ with sterile PBS, and then immersed in a $3 \mathrm{mg} / \mathrm{mL}$ sulfo-SMCC solution for 2 hours. The scaffold was then rinsed $(\times 3)$ and biofunctionalised with LTGF as de- 
Fig. 2. A schematic illustration of scaffold biofunctionalisation techniques

Electrospun PLLA scaffolds are plasma treated and then functionalised with LTGF by two methods:random and orientated approaches. PLLA, poly(L-lactide); TGF, transforming growth factor; sulfo-SMCC, sulfosuccinimidyl-4-( $\mathrm{N}$-maleimidomethyl)cyclohexane-1-carboxylate; LTGF, latent transforming growth factor- $\beta 1$; pLTGF, random immobilisation of latent TGF- $\beta 1$ on plasma treated PLLA scaffold; sLTGF, orientated immobilisation of latent TGF- $\beta 1$ using sulfo-SMCC on plasma treated PLLA scaffold.

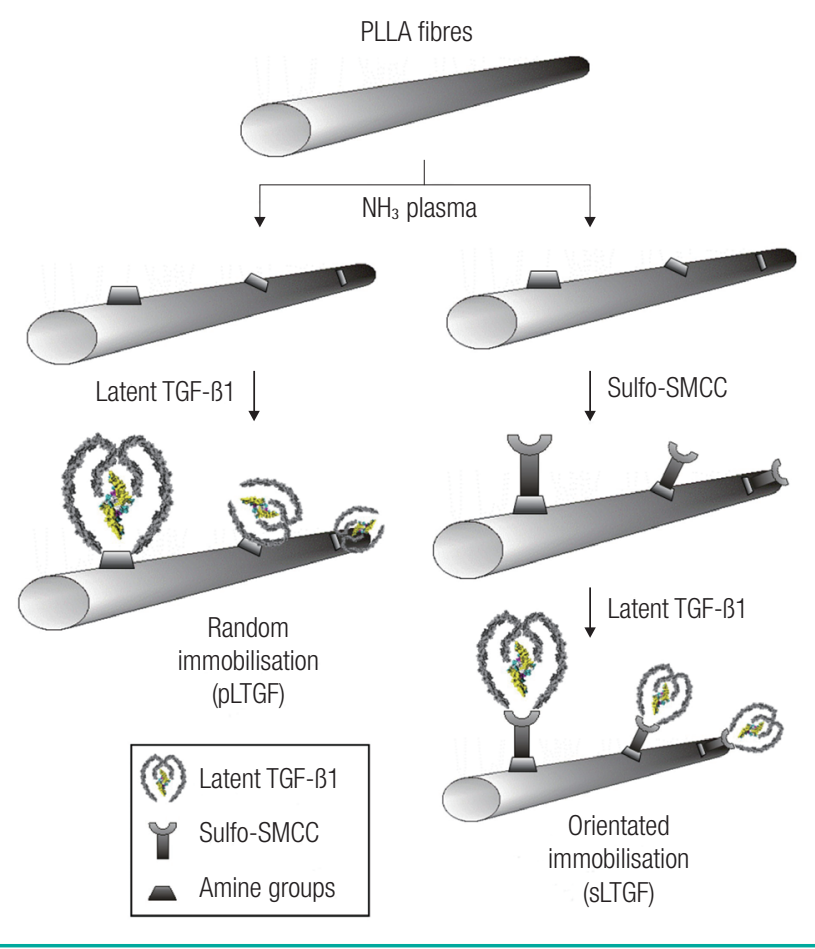

scribed for random immobilisation.

\section{Characterisation of biofunctionalised scaffolds}

Structural characterisation with scanning electron microscopy All of the samples were sputter coated with gold (Emitech K550, Quorum Technologies, Kent, UK) and observed under scanning electron microscopy (FESEM, Gemini LEO1525) at an accelerating voltage of $4 \mathrm{kV}$.

\section{Surface chemistry analysis using XPS and ninhydrin assay}

After plasma treatment, all of the scaffolds and controls were analysed with X-ray photoelectron spectroscopyb (XPS), and the corresponding surface atomic percentages of $\mathrm{N}$ were calculated as previously described [10]. Ninhydrin (Sigma, Gillingham, UK) was used to determine the quantity of primary amine groups on the ammonia plasma surface-modified scaffolds. The assay was performed as previously described [11]. The absorbance was measured at $570 \mathrm{~nm}$ on a UV-vis spectrophotometer (Lambda 25, Perkin Elmer, Chicago, IL, USA). A calibration
Table 1. Experimental groups and their sample ID

\begin{tabular}{|c|c|}
\hline Sample ID & Description \\
\hline PLLA & Untreated scaffolds \\
\hline ptPLLA+/- & Plasma treated scaffolds \\
\hline TGF & $\begin{array}{l}\text { ptPLLA with supplementation of } 10 \mathrm{ng} / \mathrm{mL} \text { active TGF- } \beta 1 \\
\text { for up to } 7 \text { days }\end{array}$ \\
\hline pLTGF+/- & ptPLLA with LTGF random immobilisation \\
\hline SLTGF+/- & ptPLLA with LTGF orientated immobilisation \\
\hline
\end{tabular}

For the in vivo study, the symbols (+) or (-) indicate scaffolds seeded with cells and their cell-free controls, respectively.

ID, identification; PLLA, poly(L-lactide).

curve was obtained using glycine standards.

Quantification of latent TGF- $\beta 1$ on scaffolds using an immunoassay

The quantification of the immobilised LTGF was performed on biofunctionalised scaffolds and compared to controls (Table 1) using a method described previously [3] to enzymatically cleave the LTGF off the scaffold. Subsequently, quantification of active TGF- $\beta 1$ was performed using a Quantikine Human TGF- $\beta 1$ Immunoassay (R\&D Systems, Abingdon, UK) as per the manufacturer's protocol.

\section{Biological characterisation Cell culture}

Human primary nasal septal chondrocytes were provided by Dr. R. Ghazanfar (RREC3852, Imperial College London, UK). The chondrocytes were expanded to the third passage in chondrocytic growth media (CGM) [12] and seeded at 9,000 cells/ $\mathrm{cm}^{2}$ onto each scaffold (Table 1) in serum-free media (SFM) [13]. For the gene expression studies, samples were seeded at $3.125 \times 10^{4}$ cells $/ \mathrm{cm}^{2}$. Approximately $5 \times 10^{5}$ cells were snapfrozen as $\mathrm{T}_{0}$ for gene expression analysis. The CGM and SFM were replaced every 3 days. The cells cultured in the TGF group received an additional $10 \mathrm{ng} / \mathrm{mL}$ of human recombinant TGF- $\beta 1$ (R\&D Systems) supplementation for up to 7 days. The cell-scaffold constructs were collected at 1,7 , and 14 days for evaluation of cell viability and gene expression. In the in vivo study, chondrocytes were taken to the fourth passage in CGM and seeded (Table 1 ) at $8 \times 10^{5}$ cells $/ \mathrm{cm}^{2}$ in SFM. After 24 hours, the cell-scaffold constructs were implanted.

\section{Cell viability assay}

Cell viability was assessed using the LIVE/DEAD Viability/ Cytotoxicity Kit (Molecular Probes, Leiden, Netherlands). On days 1,7 , and 14, the assay was performed according to the manufacturer's protocol. 
Real-time reverse transcriptase polymerase chain reaction (RTPCR)

Total RNA extraction, reverse transcription and PCR were performed as previously described [14]. The TaqMan probes (Applied Biosystems, USA) are detailed in Table 2. 18S was used as a control gene. Each reaction was carried out in triplicate. For the in vivo study, frozen implants were homogenised in $350 \mu \mathrm{L}$ RLT buffer, using TissueLyser II (both Qiagen, Manchester, UK) operated at $30 \mathrm{~Hz}$ for two 3-minutes cycles prior to RNA extraction. The mRNA expression was calculated using the comparative $\mathrm{C}_{\mathrm{T}}$ method [15]. Fold changes in gene expression are presented as mean \pm standard deviation relative to day 0 cells.

\begin{tabular}{|c|c|c|c|}
\hline Gene name & Symbol & Catalogue no. & Accession no. \\
\hline $\begin{array}{l}\text { Sex-determining region } \\
\text { Y-box } 9\end{array}$ & Sox9 & Hs00165814 & NM000346 \\
\hline Collagen type 1 & Col1A1 & Hs00164004 & NM000088 \\
\hline Collagen type 2, alpha 1 & Col2A1 & Hs00156568 & NM001844.4 \\
\hline Eukaryotic 18S rRNA & $18 \mathrm{~S}$ & Hs99999901 & X03205 \\
\hline
\end{tabular}

\section{In vivo implantation of cell-scaffold constructs}

Cell-scaffold constructs and their no-cell controls were implanted into individual dorsal subcutaneous pockets of four 5- to 6-week-old female athymic rats $(r n u / r n u$, Harlan, Bicester, UK) under a UK Home Office approved project licence (PPL70/6600). The approximate anatomical landmarks of the various scaffold groups are illustrated in Fig. 3. The animals were maintained according to institutional guidelines. After 6 weeks, all of the rats were sacrificed and all of their implants were retrieved immediately. Each implant was divided equally for the following investigations.

\section{Cryosection of implants}

The implants were embedded in optimum cutting temperature embedding compound (Bright Cryo-M-Bed, Bright Instrument Company Ltd., Huntingdon, UK). Full depth sections of 7- $\mu \mathrm{m}$ thickness from all of the scaffold groups were cut using a cryostat (Bright Instrument Company Ltd.) and fixed in ice-cold acetone for 5 minutes. The sections were then submitted for SEM, histology and immunofluorescence analyses as described below.

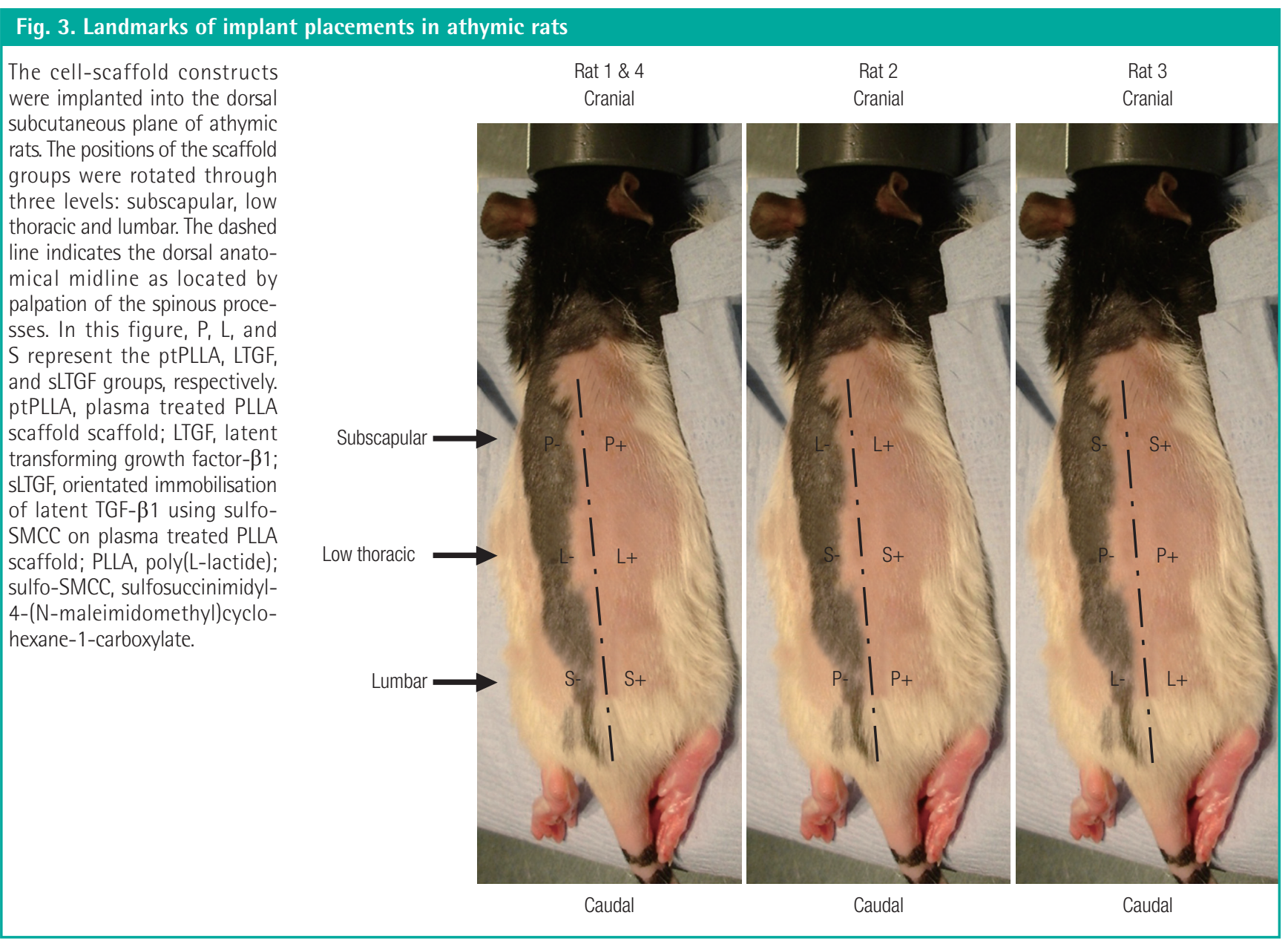


Histology and immunofluorescence analyses of implants The sections were stained with 1 ) haematoxylin (Gill's Formula, Vector Laboratories, Peterborough, UK) and eosin (SigmaAldrich, Gillingham, UK) to evaluate general tissue-implant morphology and cellular organisation and 2) $0.001 \%(\mathrm{w} / \mathrm{v})$ fast green, $0.1 \%(\mathrm{w} / \mathrm{v})$ safranin $\mathrm{O}$ (Sigma-Aldrich) and haematoxylin to assess GAG distribution.

All of the sections were blocked with 2\% (w/v) BSA and 0.2\% Triton-X100. Primary and secondary antibodies were incubated in a humidified chamber at $37^{\circ} \mathrm{C}$ for 1 hour. To reveal the presence of donor cells, sections were incubated with a mouse monoclonal antibody specific to a human nuclear antigen (Millipore, Watford, UK) at a 1:50 dilution with $2 \%(\mathrm{w} / \mathrm{v})$ BSA. For detection of human type I and II collagen production, sections were incubated with rabbit anti-collagen I polyclonal and mouse anti-collagen II monoclonal antibodies (1:80 and 1:100 dilutions, respectively; both from Abcam, Cambridge, UK). Primary antibodies were incubated separately with 1) Texas Red conjugated sheep polyclonal to rabbit antibody for the detection of collagen I (596-620 nm) and 2) diaminotriazinylaminofluorescein (DTAF)-conjugated goat monoclonalto-mouse antibody for the detection of human nuclei and collagen II (1:1000 dilution, Abcam) (495-528 nm). Vectashield mounting medium containing 4'6-diamindino-2-phenylindole (DAPI) (Vector Laboratories) was added to reveal all of the DNA (358-461 nm). The experiments were performed on four samples with triplicate sections.

\section{Statistical significance}

The means and standard deviations of the results were calculated using the SPSS ver. 12.0 (SPSS Inc., Chicago, IL, USA). The Kruskal-Wallis test for several independent samples and Mann-
Whitney U test for two independent samples were performed to determine the statistical significance of differences among various scaffolds. A P-value of $<0.05$ was considered to be significant.

\section{RESULTS}

\section{Structural architecture of electrospun scaffolds}

SEM analyses revealed that the electrospun PLLA scaffolds were uniform, randomly orientated, and closely resembled the architecture of the cartilage ECM, with a fibre diameter of $242 \pm 58$ nm (Fig. 4).

\section{Surface chemistry analysis using XPS and ninhydrin as- say \\ Surface chemical analysis using XPS demonstrated that nitrog- enous species were incorporated onto the scaffold surfaces after plasma treatment. Both XPS spectra (Fig. 5) and surface elemental compositional analysis (Table 3 ) revealed that the in- tensity $(400.0 \mathrm{eV})$ and amount of nitrogenous species increased proportionally with exposure time and power. The highest quantity of surface nitrogenous species was detected on plasma- treated scaffolds when the plasma treatment was $100 \mathrm{~W}$ for 10 minutes ( $5.2 \pm 0.3$ atomic \%). Consistently, the ninhydrin assay demonstrated that the density of primary amine groups on the scaffold surfaces increased with longer exposure time and higher power during plasma treatment (highest $66.4 \pm 4.3 \mathrm{nmol} / \mathrm{mg}$ PLLA at $100 \mathrm{~W}, 10$ minutes).}

\section{Quantification of latent TGF- $\beta 1$ on scaffolds using im- munoassay}

The results of the TGF- $\beta 1$ immunoassay showed that the LTFG

\section{Fig. 4. Micro-structural architecture of the electrospun scaffold}

This figure illustrates the structural resemblance between the architecture of native human hyaline cartilage (A) and the electrospun PLLA scaffold (B). The SEM image of native cartilage (A) was fixed in 2.5\% glutaraldehyde, followed by stepwise dehydration with critical point drying and sputtered gold coating as mentioned above (scale bar $=2 \mu \mathrm{m})$. PLLA, poly(L-lactide); SEM, scanning electron microscopy.
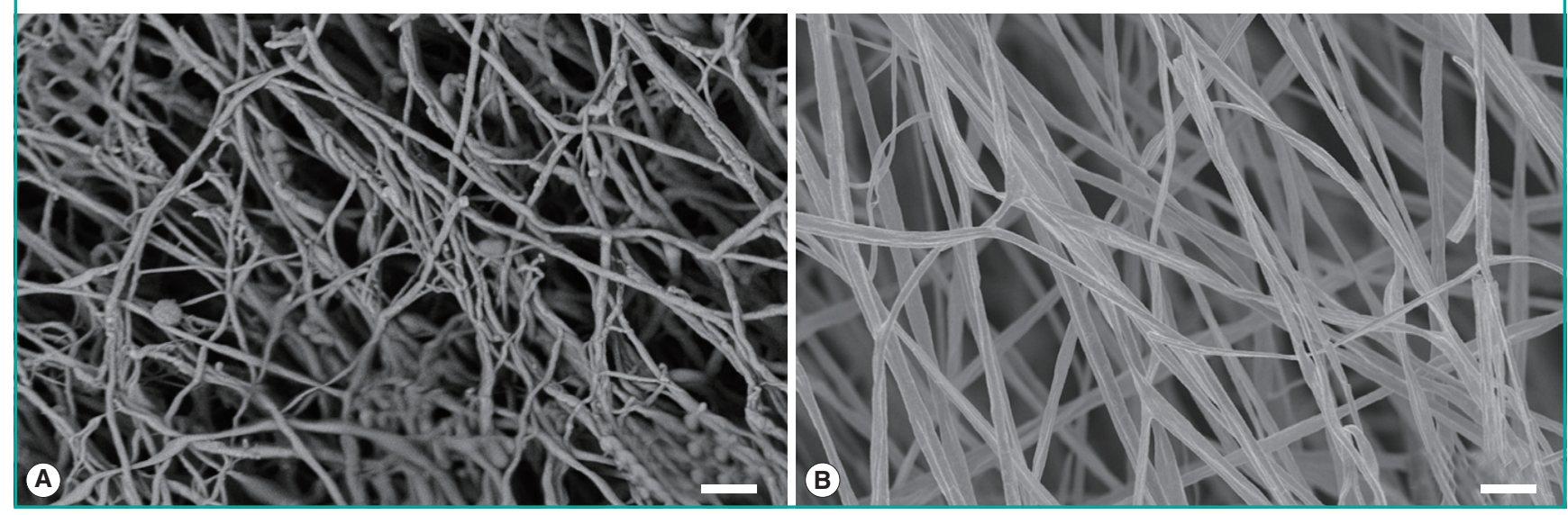
was successfully grafted onto the scaffold surfaces, using both random and orientated LTGF immobilisation techniques. An average of $195.4 \pm 4.3 \mathrm{pg} / \mathrm{cm}^{2}$ of TGF- $\beta 1$ was released from the pLTGF scaffolds. An average of $14.1 \pm 1.7 \mathrm{pg} / \mathrm{cm}^{2}$ of TGF- $\beta 1$

\section{Fig. 5. Surface chemistry analysis with XPS}

This figure shows the XPS spectra of ammonia plasma surfacemodified PLLA scaffolds at various exposure powers (W) and times (min). (A) Non-plasma-treated control, (B) 50 W 2 minutes, (C) 50 W 5 minutes, (D) 50 W 10 minutes, (E) 100 W 2 minutes, (F) 100 W 5 minutes, (G) $100 \mathrm{~W} 10$ minutes. XPS, X-ray photoelectron spectroscopy; PLLA, poly(L-lactide); CPS, counts per second.

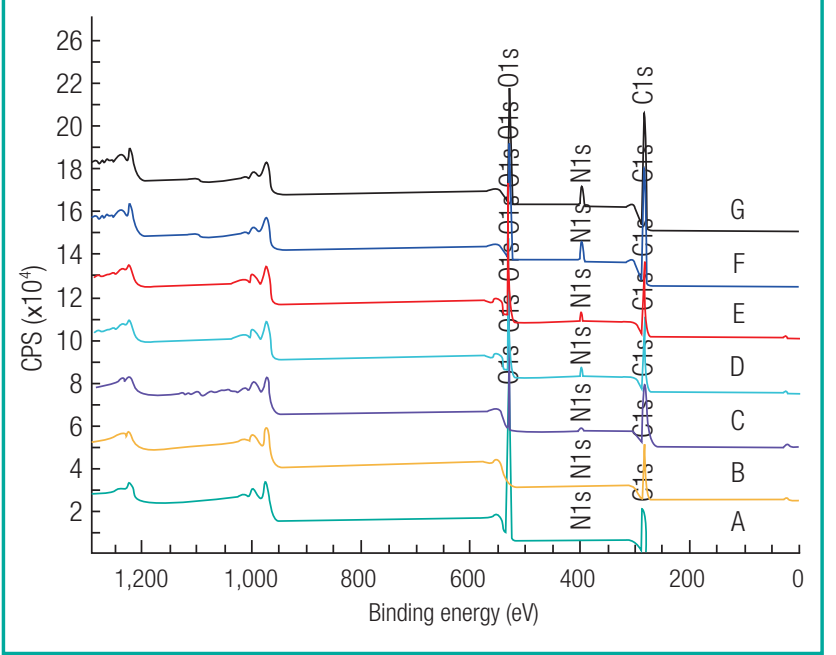

was released from the sLTGF scaffolds. No TGF- $\beta 1$ was detected on the control scaffolds.

\section{Effects of various scaffolds on cell viability assay}

The LIVE/DEAD assay on days 1, 7, and 14 showed a high live-to-dead ratio across experimental groups indicating that all of the plasma-treated scaffolds were non-cytotoxic to primary human chondrocytes and maintained cell viability for 7 days ( $>80 \%$ ) (Fig. 6). At day 14, cell viability had declined in all of the experimental groups to the range of $70 \%$ to $80 \%$ except for the TGF group (57.7\%).

Table 3. Amount of nitrogen and primary amine groups detected on the surface-modified scaffolds at various plasma treatment powers $(\mathrm{W})$ and exposure times $(\mathrm{min})$

\begin{tabular}{|c|c|c|}
\hline & Atomic \% (N 1s) & Amount of $\mathrm{NH}_{2}\left(\mathrm{nmol} / \mathrm{mg}\right.$ PLLA) ${ }^{\mathrm{b})}$ \\
\hline PLLA control & 0.0 & 0.0 \\
\hline $50 \mathrm{~W}-2 \mathrm{~min}$ & $0.6 \pm 0.1$ & $27.9 \pm 0.3$ \\
\hline $50 \mathrm{~W}-5 \mathrm{~min}$ & $1.2 \pm 0.2$ & $33.2 \pm 0.6$ \\
\hline $50 \mathrm{~W}-10 \mathrm{~min}$ & $3.0 \pm 0.1$ & $37.9 \pm 0.8$ \\
\hline $100 \mathrm{~W}-2 \mathrm{~min}$ & $2.9 \pm 0.2$ & $44.8 \pm 1.2$ \\
\hline $100 \mathrm{~W}-5 \mathrm{~min}$ & $4.2 \pm 0.3$ & $57.8 \pm 1.4$ \\
\hline $100 \mathrm{~W}-10 \mathrm{~min}$ & $5.2 \pm 0.3$ & $66.4 \pm 4.3$ \\
\hline \multicolumn{3}{|c|}{$\begin{array}{l}\text { PLLA, poly(L-lactide). } \\
\text { a)Determined by } X \text {-ray photoelectron spectroscopyb; b) Determined by ninhydrin } \\
\text { assay. }\end{array}$} \\
\hline
\end{tabular}

\section{Fig. 6. Cell viability of human chondrocyte culture}

The cell viability of primary human chondrocytes cultured on various scaffold types at 1, 7, and 14 days is shown (scale bar $=200 \mu \mathrm{m}$ ). The numbers shown in each image indicate the mean percentage \pm standard deviation. The average number of cells per image on day 1, 7 and 14 were approximately 30,50, and 30, respectively. PLLA, untreated poly(L-lactide) scaffold; ptPLLA, plasma treated PLLA scaffold; TGF, ptPLLA with supplementation of $10 \mathrm{ng} / \mathrm{mL}$ active TGF- $\beta 1$ for up to 7 days; pLTGF, random immobilisation of latent TGF- $\beta 1$ on plasma treated PLLA scaffold; sLTGF, orientated immobilisation of latent TGF- $\beta 1$ using sulfo-SMCC on plasma treated PLLA scaffold; sulfo-SMCC, sulfosuccinimidyl-4-(Nmaleimidomethyl)cyclohexane-1-carboxylate.
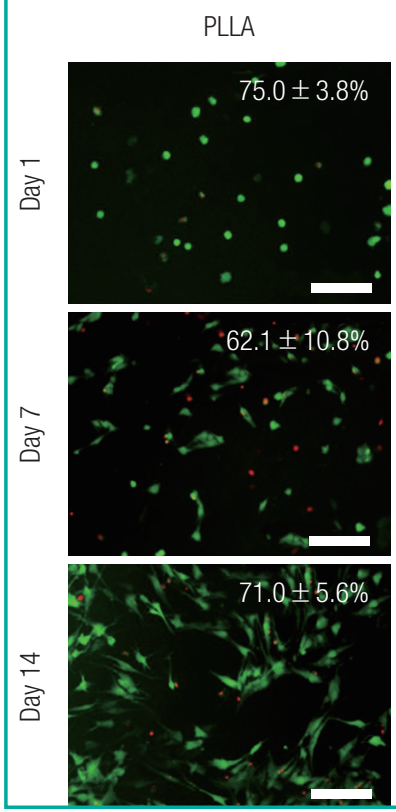

ptPLLA
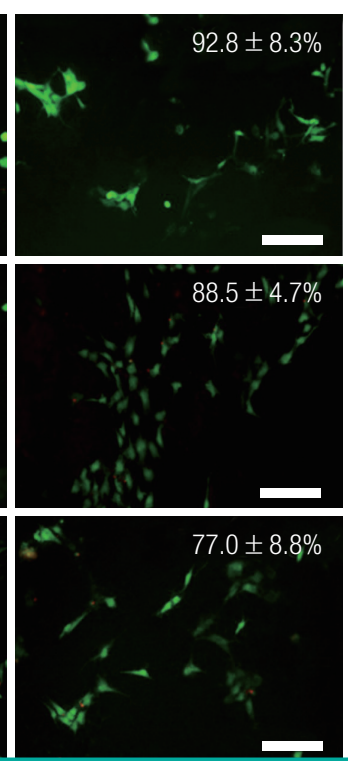

TGF
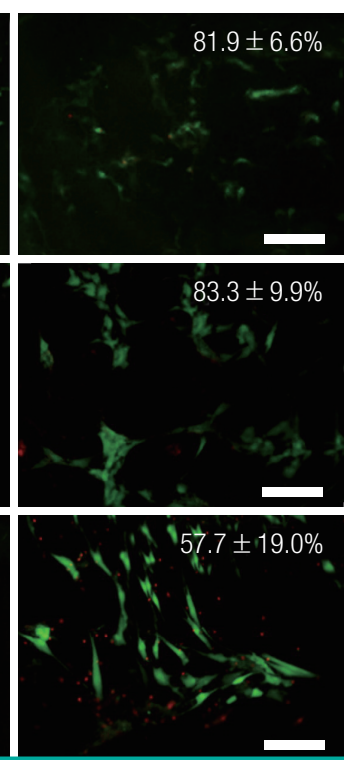

pLTGF
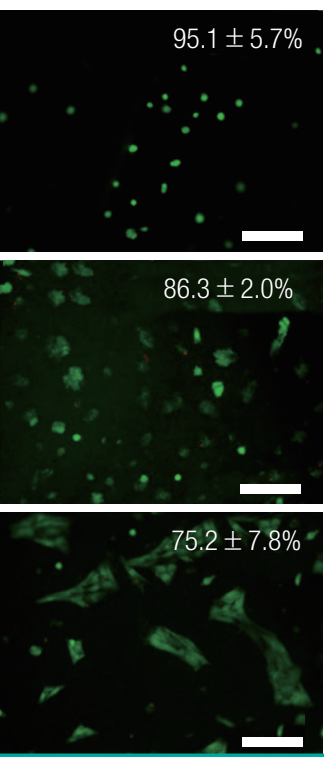

SLTGF

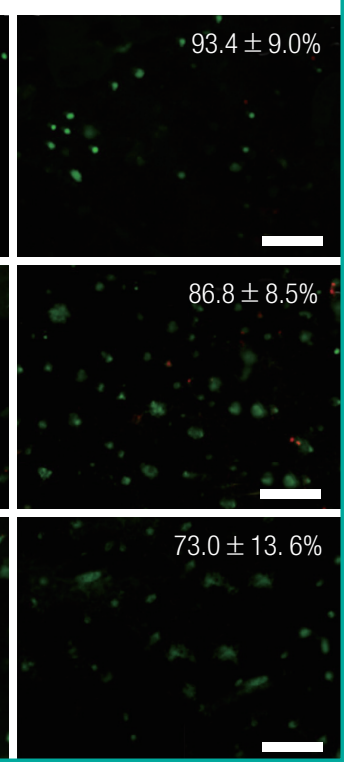


Effects of various scaffolds on gene expression of in vitro cultured cell-scaffold constructs

The results showed that the scaffold groups significantly influenced both Sox9 and Col1A1 gene expression (Kruskal-Wallis test, $\mathrm{P}<0.05$ ). The pLTGF group significantly up-regulated the Sox 9 expression by approximately 10 -fold when compared to day 0 . This level of expression was also significantly higher than those of all of the other scaffold groups in this experiment (Fig. 7). CollA1 was significantly higher in the TGF group, as compared to the other scaffold groups. Col2A1 was not detected in any of the groups.

Effects of various scaffolds on gene expression analysis of in vivo cultured implants

The results showed that the scaffold groups significantly influenced both Sox9 and Col2A1 gene expression (Kruskal-Wallis test, $\mathrm{P}<0.05)$ but not that of Col1A1. Sox 9 was expressed across all experimental groups (Fig. 8A). The Sox9 expression observed in the pLTGF+ groups was approximately 1.2-fold and 3.9-fold higher when compared to the ptPLLA+ and sLTGF+ groups, respectively. Col2A1 was expressed in the PLTGF+ and SLTGF+ groups, while it was undetectable in ptPLLA+ (Fig. 8B). Col1A1 expression was detected in all of the scaffold groups with no significant differences among any of the groups (Fig. 8C).

\section{Fig. 7. Effects of scaffolds on chondrocyte gene expression}

This figure illustrates the mRNA expression profiles of chondrocytes cultured on various scaffold types for 14 days. The data were analysed with real-time reverse transcription-polymerase chain reaction. The Sox9 expression in the pLTGF group, indicated by $\left({ }^{*}\right)$, was significantly higher than that of the other groups (Mann-Whitney $\mathrm{U}$ test, $\mathrm{P}<0.05$ ). In the TGF group, Col1A1 and Sox9 expression, represented by the symbols $(\bullet)$ and $(\diamond)$, respectively, were significantly higher $(P<0.05)$ than those of the other groups, except Sox9 expression in the pLTGF group. The results are presented as average change \pm standard deviation of repeated experiments $(n=3)$. PLLA, untreated poly $(L-$ lactide) scaffold; ptPLLA, plasma treated PLLA scaffold; TGF, ptPLLA with supplementation of $10 \mathrm{ng} / \mathrm{mL}$ active TGF- $\beta 1$ for up to 7 days: pLTGF, random immobilisation of latent TGF- $\beta 1$ on plasma treated PLLA scaffold; sLTGF, orientated immobilisation of latent TGF- $\beta 1$ using sulfo-SMCC on plasma treated PLLA scaffold.

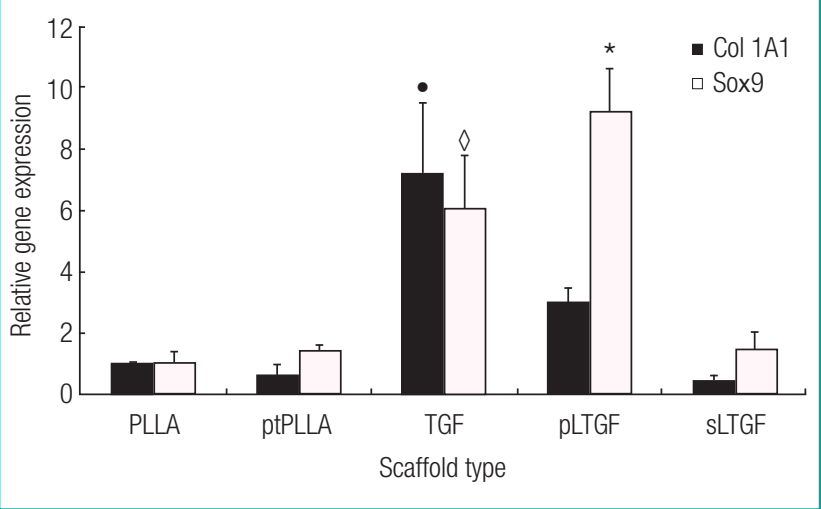

Scanning electron microscopy, histology and immunofluorescent staining of in vivo implant sections

SEM images of the implant sections indicated that the tissue products were integrated into the scaffolds (Fig. 9A-F). Hae-

\section{Fig. 8. Gene expression of in vivo cultured chondrocytes}

This figure reveals the effects of various scaffold types on in vivo gene expression of chondrocytes at six weeks. Expression levels of (A) Sox9, (B) Col2A1, and (C) Col1A1 were normalised to the $18 \mathrm{~S}$ gene and day 0 expression levels. The average fold changes \pm standard deviation are presented $(n=4)$. The following symbols represent significant differences (Mann-Whitney $\mathrm{U}$ test, $\mathrm{P}<0.05)$ in the $\left(^{*}\right)$ ptPLLA+ and $(\bullet)$ sLTGF+ groups. PLLA untreated poly(L-lactide) scaffold; ptPLLA, plasma treated PLLA scaffold; pLTGF, random immobilisation of latent TGF- $\beta 1$ on plasma treated PLLA scaffold; sLTGF, orientated immobilisation of latent TGF- $\beta 1$ using sulfo-SMCC on plasma treated PLLA scaffold.
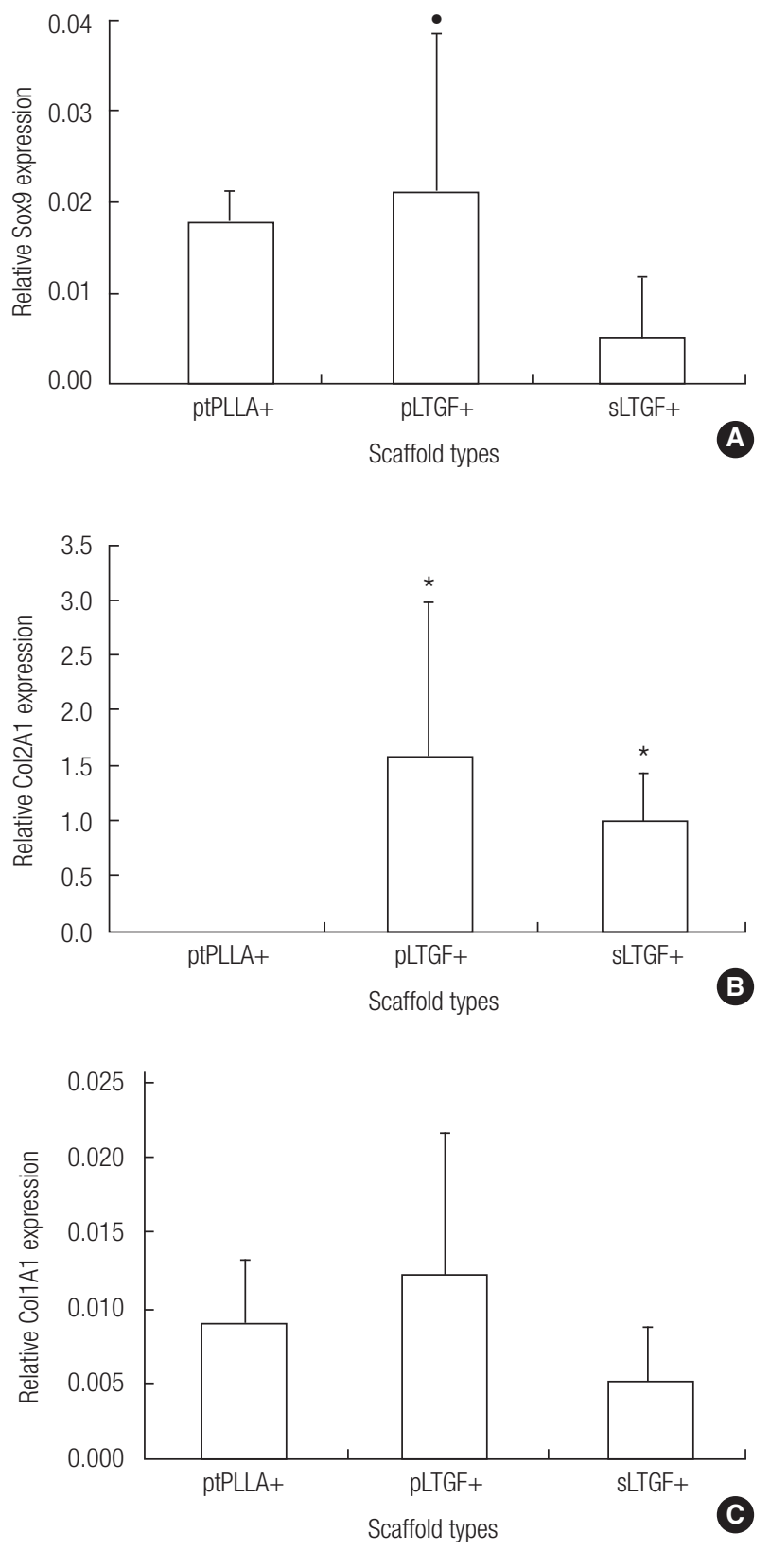
matoxylin and eosin ( $\mathrm{H} \& \mathrm{E})$ examination revealed that all of the scaffolds were integrated with the surrounding rat tissue on both surfaces of the scaffolds (Fig. 9G-I). Cells could be found within the thickness of each scaffold. At 6 weeks, the donor human chondrocytes were detected adjacent to the scaffolds (Fig. 9J-O). Histological staining with safranin $\mathrm{O}$ was negative (results not shown). Human type II collagen was detected in the pLTGF+ group only (Fig. 9Q), while type I collagen was found in the ptPLLA+ scaffold (Fig. 9S).

\section{DISCUSSION}

TGF- $\beta 1$ has been used extensively to control growth and differ- entiation in tissue engineering, and a variety of delivery systems have been explored. The concept behind the scaffold system developed in this study is to guide cell-mediated activation. This may allow cells to determine their proliferation and function as necessary, preventing potential TGF- $\beta 1$ adverse effects. LTGF was incorporated into a novel biomimetic regenerative scaffold relevant to cartilage engineering. Our results confirm that an LTGF biofunctionalised scaffold design successfully influenced chondrocytic behaviour based on Sox 9 and Col2A1 expression in vitro and in vivo.

The TGF- $\beta 1$ immunoassay revealed that the LTGF was successfully anchored onto the plasma treated scaffold surface. The random immobilisation technique was more efficient in an-

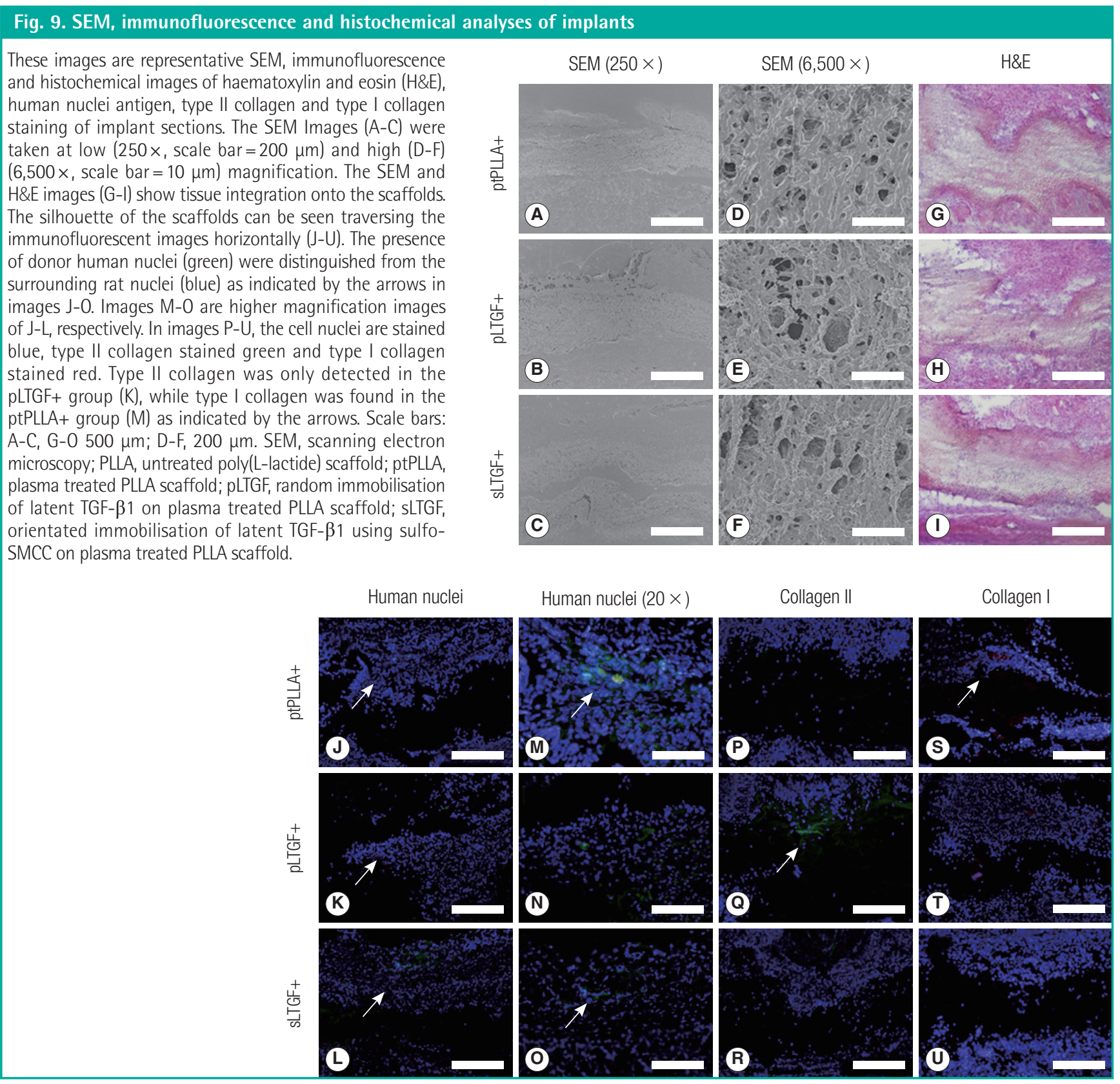


choring a larger amount of the LTGF (approximately one order of magnitude), as compared to the orientated immobilisation approach. This could be for the following reasons: 1) the LAP has limited free thiol groups; 2) the specific location of the free thiols of the LAP may be sterically hindered; and 3) the short spacer contained in the sulfo-SMCC further limits its scope of reaction with distant thiol groups. The immunoassay also demonstrated that the immobilised LTGF remains available for enzymatic and chemical activation. The bioavailability of the TGF- $\beta 1$ on the LTGF biofunctionalised scaffolds was further confirmed by primary human chondrocytic culture.

The results of the LIVE/DEAD assay demonstrated that the LTGF biofunctionalised scaffolds are non-cytotoxic and can sustain cell viability of over $70 \%$. The TGF group, despite receiving TGF- $\beta 1(10 \mathrm{ng} / \mathrm{mL})$ supplementation for up to 7 days, failed to sustain the level of cell viability seen in the LTGF biofunctionalised groups. The general declining trend in cell viability over time may be related to the use of SFM. A serum-free environment is used to revert the mitotic cell cycle (proliferation) induced by serum into the interphase cycle (differentiation) [16]. In native ECM, cells can only express a specific function while they remain in the interphase, and chondrocytes naturally arrest in an infinite interphase cycle [16]. Once isolated from their natural environment and passaged in vitro, dedifferentiation occurs-classically characterised by the down-regulation of cartilage-specific genes (e.g., Sox9 and Col2A1) and a gradual rise in CollA1 (dedifferentiation marker) [17]. The ability to redifferentiate declines with the passage number. Sox 9 is a key transcription factor in chondrogenic differentiation that directly up-regulates the expression of Col2A1 [18]. Its expression occurs in all chondroprogenitor cells and chondrocytes, except hypertrophic chondrocytes, and has been reported to parallel Col2A1 during embryonic chondrogenesis [19].

In this study, primary human chondrocytes were cultured up to the third and fourth passages. At such passage numbers, a dedifferentiated phenotype is expected, and Col2A1 was undetectable at 14 days of in vitro culture. This was consistent with studies which have shown that passaged chondrocytes only reexpressed the Col2A1 gene after 21 days of in vitro SFM culture [13]. The first indication of redifferentiation at 14 days was demonstrated in the pLTGF and TGF groups with significantly up-regulated cartilage-specific transcription factor Sox9. The upregulation of Sox9 seen in the TGF group was consistent with a previous study [20]. Interestingly, the up-regulation of Sox9 in the pLTGF group was significantly higher (approximately 30\%) when compared to the TGF group, considering the amount of LTGF available from the pLTGF scaffold $\left(195.4 \mathrm{pg} / \mathrm{cm}^{2}\right.$ scaffold)-approximately 150-fold lower than the standard TGF- $\beta 1$ supplementary dose received by the TGF group $(10 \mathrm{ng} / \mathrm{mL})$ [21]. This suggests that although the anchorage of the LTGF onto the pLTGF scaffold may not achieve the intended natural configuration, the LTGF remained protected and available for activation. Although TGF- $\beta 1$ is known to have positive effects on matrix production and chondrocytic differentiation [1], some studies have reported its suppressive effects on collagen type II synthesis and the down-regulation of chondrocyte differentiation [22]. The high Col1A1 expression in the TGF group is consistent with such studies. The sLTGF group did not significantly up-regulate Sox 9 gene expression. This may reflect the relatively small concentration of TGF- $\beta 1$ present in the sLTGF scaffold.

At the end of the 6 weeks of in vivo study, the survival of the donor human cells was confirmed by the detection of humanspecific genes by reverse transcription-polymerase chain reaction and anti-human nuclei immunohistochemistry. Of significance, Col2A1 expression was detected on the pLTGF+ and sLTGF+ groups. This confirms that the dedifferentiated chondrocytes are induced to redifferentiate by the LTGF biofunctionalised scaffolds. The expression of Col2A1 was undetectable in the implanted controls (ptPLLA+) despite the presence of Sox9. Although Sox9 is required for the activation of the Col2A1 gene, the results demonstrate that Sox 9 expression in the ptPLLA+ group is not sufficient in activating the Col2A1 gene in the absence of the LTGF biofunctionalised scaffolds.

Although Sox9 expression in the sLTGF+ group was significantly lower than in the ptPLLA+ group, Col2A1 was expressed. The level of Sox 9 expression seen in the pLTGF+ group, which was 3.9-fold higher than that of sLTGF+, was not accompanied by a proportional rise in Col2A1 expression when compared to the sLTGF+ group. This "anti-parallel" trend between Col2A1 and Sox 9 may be a gene profile characteristic of in vitro passaged adult human chondrocytes, where the expression of Sox 9 correlates to a lesser extent with the presence of Col2A1 [18]. This may be due to a decrease in Sox 9 binding activity to the Col2A1 gene enhancer site with increasing passage number [18].

Chondrogenesis in the LTGF biofunctionalised groups was evident in the immunofluorescence detection of type II collagen. Human type II collagen was detected in sections of the pLTGF+ group to further confirm the chondrogenic efficacy of the biofunctionalised scaffolds (Fig. 9).

Although integration can be seen on the H\&E stains and SEM images (Fig. 9), negative staining for glycosaminoglycan with safranin $\mathrm{O}$ (results not shown) might reflect a slow redifferentiation of the dedifferentiated chondrocytes [23]. In addition, TGF- $\beta 1$ may reduce glycosaminoglycan synthesis in mature chondrocytes and increase the degradation of aggrecan by 
up-regulating aggrecanase activity [24]. This may explain the negative staining of safranin $\mathrm{O}$ in all of the scaffold groups. The type I collagen on immunofluorescence, found in the ptPLLA+ group, was consistent with CollA1 gene expression and lack of Col2A1 expression-and further confirmed that those chondrocytes remained in their de-differentiated state.

We can deduce that in such systems there are two key mechanisms by which TGF- $\beta 1$ influences chondrocyte behaviour: firstly, by dose, and secondly, by factor presentation (active vs. latent form). A high dose and frequent supplementation of active growth factor may not necessarily result in the desired response (as seen in the low Sox 9 and up-regulation of CollA1 in the TGF group). Conversely, a dose 150-fold lower, coupled with the presentation of the growth factor in its latent form to cells, resulted in a change in the pattern of gene expression of the chondrocytes. Further LTGF dose titration and protein conformational studies need to be performed to verify this argument.

Numerous studies have "immobilised" or "incorporated" growth factors such as TGF- $\beta 1$ and fibroblast growth factor into tissue scaffold systems with sustained release properties [25]. These studies have used active forms and inevitably involved processing steps which may have resulted in reduced bioactivity. In addition, the majority of scaffold release systems are hydrogel based, and release of growth factors is by diffusion or degradation of the scaffold matrix. This often results in a "burst" release and a compromise in scaffold mechanical and other properties required for cartilage engineering [1]. In our strategy, the growth factor remains immobilised on the scaffold and protected by its latent protein until cell activation.

Scaffolds functionalised with a latent form of TGF- $\beta 1$ have been used here to direct cell-mediated activation of this key growth factor in chondrogenesis. The presence of the growth factor influenced the chondrocyte cell phenotype, and this strategy may well prove useful for other growth factors and more widespread application in the field of tissue engineering.

\section{ACKNOWLEDGEMENTS}

The authors would like to thank Ms. Emily Smith for technical assistance and data processing on XPS surface characterisation at Nottingham Nanoscience and Nanotechnology Centre (NNNC) at Nottingham University, EPSRC for open access materials analysis at the NNNC, and EPSRC Challenging Engineering Grant and ERC Grant Naturale, Fundação para a Ciência e a Tecnologia, The Healing Foundation and The British Association of Plastic Reconstructive and Aesthetic Surgeons for their financial support.

\section{REFERENCES}

1. Sohier J, Moroni L, van Blitterswijk C, et al. Critical factors in the design of growth factor releasing scaffolds for cartilage tissue engineering. Expert Opin Drug Deliv 2008;5:543-66.

2. Guerne PA, Sublet A, Lotz M. Growth factor responsiveness of human articular chondrocytes: distinct profiles in primary chondrocytes, subcultured chondrocytes, and fibroblasts. J Cell Physiol 1994;158:476-84.

3. Pedrozo HA, Schwartz Z, Gomez R, et al. Growth plate chondrocytes store latent transforming growth factor (TGF)-beta 1 in their matrix through latent TGF-beta 1 binding protein-1. J Cell Physiol 1998;177:343-54.

4. Annes JP, Munger JS, Rifkin DB. Making sense of latent TGFbeta activation. J Cell Sci 2003;116:217-24.

5. Saharinen J, Keski-Oja J. Specific sequence motif of 8-Cys repeats of TGF-beta binding proteins, LTBPs, creates a hydrophobic interaction surface for binding of small latent TGF-beta. Mol Biol Cell 2000;11:2691-704.

6. Koli K, Saharinen J, Hyytiainen M, et al. Latency, activation, and binding proteins of TGF-beta. Microsc Res Tech 2001; 52:354-62.

7. Wakefield LM, Winokur TS, Hollands RS, et al. Recombinant latent transforming growth factor beta 1 has a longer plasma half-life in rats than active transforming growth factor beta 1, and a different tissue distribution. J Clin Invest 1990; 86:1976-84.

8. Saharinen J, Hyytiainen M, Taipale J, et al. Latent transforming growth factor-beta binding proteins (LTBPs): structural extracellular matrix proteins for targeting TGF-beta action. Cytokine Growth Factor Rev 1999;10:99-117.

9. Mierisch CM, Cohen SB, Jordan LC, et al. Transforming growth factor-beta in calcium alginate beads for the treatment of articular cartilage defects in the rabbit. Arthroscopy 2002; 18:892-900.

10. Janssens S, Roberts C, Smith EF, et al. Physical stability of ternary solid dispersions of itraconazole in polyethyleneglycol 6000/hydroxypropylmethylcellulose 2910 E5 blends. Int J Pharm 2008;355:100-7.

11. Zhu Y, Gao C, Liu X, et al. Surface modification of polycaprolactone membrane via aminolysis and biomacromolecule immobilization for promoting cytocompatibility of human endothelial cells. Biomacromolecules 2002;3:1312-9.

12. Li WJ, Cooper JA Jr, Mauck RL, et al. Fabrication and characterization of six electrospun poly(alpha-hydroxy ester)based fibrous scaffolds for tissue engineering applications. Acta Biomater 2006;2:377-85.

13. Li WJ, Danielson KG, Alexander PG, et al. Biological re- 
sponse of chondrocytes cultured in three-dimensional nanofibrous poly(epsilon-caprolactone) scaffolds. J Biomed Mater Res A 2003;67:1105-14.

14. Ball MD, Bonzani IC, Bovis MJ, et al. Human periosteum is a source of cells for orthopaedic tissue engineering: a pilot study. Clin Orthop Relat Res 2011;469:3085-93.

15. Schmittgen TD, Livak KJ. Analyzing real-time PCR data by the comparative C(T) method. Nat Protoc 2008;3:1101-8.

16. Strehl R, Schumacher K, de Vries U, et al. Proliferating cells versus differentiated cells in tissue engineering. Tissue Eng 2002;8:37-42.

17. von der Mark K, Gauss V, von der Mark H, et al. Relationship between cell shape and type of collagen synthesised as chondrocytes lose their cartilage phenotype in culture. Nature 1977;267:531-2.

18. Kypriotou M, Fossard-Demoor M, Chadjichristos C, et al. SOX9 exerts a bifunctional effect on type II collagen gene (COL2A1) expression in chondrocytes depending on the differentiation state. DNA Cell Biol 2003;22:119-29.

19. Zhao Q, Eberspaecher H, Lefebvre V, et al. Parallel expression of Sox9 and Col2a1 in cells undergoing chondrogenesis. Dev Dyn 1997;209:377-86.
20. Kawakami Y, Rodriguez-Leon J, Izpisua Belmonte JC. The role of TGFbetas and Sox9 during limb chondrogenesis. Curr Opin Cell Biol 2006; 18:723-9.

21. Stevens MM, Marini RP, Martin I, et al. FGF-2 enhances TGF-beta1-induced periosteal chondrogenesis. J Orthop Res 2004;22:1114-9.

22. Chadjichristos C, Ghayor C, Herrouin JF, et al. Downregulation of human type II collagen gene expression by transforming growth factor-beta 1 (TGF-beta 1) in articular chondrocytes involves SP3/SP1 ratio. J Biol Chem 2002; 277:43903-17.

23. Binette F, McQuaid DP, Haudenschild DR, et al. Expression of a stable articular cartilage phenotype without evidence of hypertrophy by adult human articular chondrocytes in vitro. J Orthop Res 1998;16:207-16.

24. Moulharat N, Lesur C, Thomas M, et al. Effects of transforming growth factor-beta on aggrecanase production and proteoglycan degradation by human chondrocytes in vitro. Osteoarthritis Cartilage 2004;12:296-305.

25. Chou CH, Cheng WT, Lin CC, et al. TGF-betal immobilized tri-co-polymer for articular cartilage tissue engineering. J Biomed Mater Res B Appl Biomater 2006;77:338-48. 